This item was submitted to Loughborough's Research Repository by the author.

Items in Figshare are protected by copyright, with all rights reserved, unless otherwise indicated.

\title{
Exercise energy expenditure and postprandial lipemia in girls
}

PLEASE CITE THE PUBLISHED VERSION

http://dx.doi.org/10.1249/MSS.0b013e3182a59ab1

\section{PUBLISHER}

(c) American College of Sports Medicine

\section{VERSION}

AM (Accepted Manuscript)

\section{PUBLISHER STATEMENT}

This work is made available according to the conditions of the Creative Commons Attribution-NonCommercialNoDerivatives 4.0 International (CC BY-NC-ND 4.0) licence. Full details of this licence are available at: https://creativecommons.org/licenses/by-nc-nd/4.0/

\section{LICENCE}

CC BY-NC-ND 4.0

\section{REPOSITORY RECORD}

Tolfrey, Keith, Alex Engstrom, Caoileann Murphy, Alice E. Thackray, Robert Weaver, and Laura A. Barrett. 2019. "Exercise Energy Expenditure and Postprandial Lipemia in Girls". figshare.

https://hdl.handle.net/2134/19440. 


\section{Exercise energy expenditure and postprandial lipaemia in girls}

Keith Tolfrey ${ }^{1}$, Alex Engstrom ${ }^{1}$, Caoileann Murphy ${ }^{1}$, Alice Thackray $^{1}$, Robert Weaver ${ }^{1}$, and Laura A. Barrett ${ }^{1}$

${ }^{1}$ Paediatric Exercise Physiology Research Group, School of Sport, Exercise and Health Sciences, Loughborough University, Loughborough, LE11 3TU, UK

\section{Corresponding author:}

Dr. Keith Tolfrey

School of Sport, Exercise and Health Sciences

Loughborough University

Loughborough

LE11 3TU

UK

k.tolfrey@lboro.ac.uk

Phone +44(0)1509 226355

Fax $+44(0) 1509226301$

Running title: Exercise and postprandial lipaemia in girls

Conflicts of Interest and Source of Funding: No conflicts of interests are reported. No funding was received for this research, other than that available internally through Loughborough University. The results of the present study do not constitute endorsement by the American College of Sports Medicine. 


\section{Abstract}

Purpose: Examine the effect of 30 and 60 min of moderate intensity treadmill walking on postprandial triacylglycerol concentrations ([TAG]) in healthy girls. Methods: Eighteen 10 to 14 year old girls (mean(SD) body mass 48(11) kg; body fat 19.0(4.6)\%; peak oxygen uptake $\left(\dot{\mathrm{VO}}_{2}\right)$ 47(6) $\mathrm{mL} \cdot \mathrm{kg}^{-1} \cdot \mathrm{min}^{-1}$ ) completed three, 2 day trials in a counter-balanced crossover design, each separated by 14 days. On day one, they rested (CON), or completed $30 \min (\mathrm{EX} 30)$ or $60 \mathrm{~min}\left(\mathrm{EX60)}\right.$ of intermittent treadmill exercise at $56 \%$ peak $\dot{\mathrm{VO}}_{2}$, inducing energy expenditures of 777 and $1536 \mathrm{~kJ}$ (186 and 367 kcal) respectively. On day two, after a 12-h fast, a capillary blood sample was taken for fasting [TAG] before a high-fat milkshake (80 kJ $\mathrm{kg}^{-1}$ body mass) was consumed. Further blood samples were taken hourly over a 6-h postprandial rest period for [TAG]. ANOVA and Student's t-tests were used to analyse the data. Results: Fasting [TAG] was lower in EX60 than CON (95\% CI -0.36 to 0.04, effect size $(E S)=0.41)$ and EX30 (95\% CI -0.47 to 0.04, ES = 0.46); all group mean concentrations were low $\left(\leq 0.90 \mathrm{mmol} \cdot \mathrm{L}^{-1}\right)$. The main effect for condition revealed differences in postprandial [TAG] over time $(\mathrm{ES}=0.36)$. The EX60 total area under the [TAG] versus time curve was lower than CON (95\% CI -2.66 to -0.04, ES 0.40) and EX30 (95\% CI -2.11 to 0.15 , ES = 0.30); CON and EX30 were similar (95\% CI -1.44 to 0.71 , ES = 0.10). Conclusion: This study demonstrates that 60 , but not 30 min of moderate treadmill exercise, with a gross energy expenditure of $1536 \mathrm{~kJ}$ (367 kcal), attenuated postprandial [TAG] in girls.

\section{Keywords:}

ADOLESCENT; FEMALE; TREADMILL; TRIACYLGLYCEROL CONCENTRATION 


\section{Introduction}

Paragraph 1 While the process of atherosclerosis is initiated during childhood, the clinical symptoms of atherosclerotic disease are not evident typically until adulthood (25). Elevated plasma triacylglycerol concentrations ([TAG]) are associated with an increased risk of atherosclerosis (38). The magnitude and duration of the rise in [TAG] in the postprandial period is positively related to the progression of atherosclerosis (4). Furthermore, prospective cohort studies have demonstrated recently that non-fasting (postprandial) [TAG] independently predicts future cardiovascular events in men and women $(1,27)$. Indeed, accumulating evidence shows that postprandial [TAG] may better predict cardiovascular disease risk than traditional fasting measures (1). Since most waking hours are postprandial, a combination of low physical activity and diets high in fat or carbohydrate may increase the risk of future cardiovascular disease with prolonged elevated [TAG] (28). Consequently interventions that modulate postprandial lipaemia by improving TAG metabolism should begin ideally during childhood and adolescence (25).

Paragraph 2 Many studies have shown that acute aerobic exercise performed up to $18 \mathrm{~h}$ before a high fat meal attenuates postprandial [TAG] in adults, resulting typically in a 15 to $25 \%$ reduction in the total area under the [TAG] versus time curve (28). In contrast, only five studies that we are aware of have examined the influence of a single bout of moderate to vigorous intensity exercise on postprandial [TAG] in adolescent boys (2, 21, 30, 34, 36). In each of these studies, the postprandial TAG response was lower after exercise compared with a non-exercise control condition. Exercise-induced energy expenditure (EE) appears to reduce postprandial [TAG] in a dose-dependent manner in adults (12, 37). In contrast, this has not been shown in studies in boys $(34,36)$. In the most recent of these studies, both 30 and 60 min of intermittent treadmill exercise at $55 \%$ of peak $\dot{\mathrm{V}} \mathrm{O}_{2}$ were shown to be similarly 
efficacious at reducing mean postprandial [TAG] in 13-year-old boys (34). Although gross $\mathrm{EE}$ in the 60 min exercise condition (1967 kJ) was approximately double that for $30 \mathrm{~min}$ (982 $\mathrm{kJ}$ ), the extra attenuation of postprandial [TAG] was only $4 \%$, leading the authors to conclude a dose-response was not evident.

Paragraph 3 Although accumulating evidence indicates that an exercise-induced reduction in postprandial [TAG] occurs in boys, it has not been possible to find any similar studies with girls. Studies have consistently demonstrated greater postprandial [TAG] in men than women $(7,16)$, which may reflect between sex differences in abdominal visceral adipose tissue, enhanced skeletal muscle clearance of circulating TAG and greater suppression of upperbody subcutaneous adipose tissue lipolysis in women $(7,15,18)$. Although the majority of exercise postprandial studies are with males, Gill et al. (13) compared 30 men and 43 women and reported that the exercise-induced attenuation in the postprandial TAG response was not different (23.5 vs. 19.8 \% respectively). However, it is not known whether exercise reduces postprandial [TAG] in girls suggesting this is a serious gap in our understanding of this important marker of future atherogenic risk in young people.

Paragraph 4 International physical activity recommendations for children and adolescents generally range from 60 to 90 min per day $(8,17)$ Evidence suggests that girls are less likely than boys to meet these recommendations (29). Furthermore, the decline in physical activity with age is more marked among young females (26). Clearly, in order for exercise intervention strategies to be effective in the long-term, the recommended exercise programmes must be considered acceptable and sustainable by the target group. This highlights the need to identify the lowest dose of exercise EE that can reduce postprandial [TAG] in girls. 
Paragraph 5 Therefore, the aim of this study was to examine the effect of 30 and 60 min of moderate intensity exercise on postprandial [TAG] in healthy, but not endurance trained, girls for the first time.

\section{Methods}

\section{Participants}

Paragraph 6 After approval from the University Ethical Advisory Committee, 18 girls volunteered for the study and provided their written assent. Written informed consent was also obtained from a parent or guardian. All participants were physically active generally, in good overall health, not taking any medications or dietary supplements known to affect lipid or carbohydrate metabolism and had no contradictions to exercise participation. Physical and physiological characteristics are presented in Table 1.

\section{Anthropometry and physical maturation}

Paragraph 7 All anthropometric measurements were conducted with participants wearing shorts, T-shirt and socks. Stature was measured to the nearest $0.01 \mathrm{~m}$ using a fixed stadiometer (Holtain, Crosswell, UK). Body mass was quantified to the nearest $0.1 \mathrm{~kg}$ using a balance-beam scale (Avery, Birmingham, UK). Body mass index (BMI) was calculated as body mass (kg) divided by stature (m) squared. Skinfold thickness was measured to the nearest 0.2 mm using Harpenden callipers (John Bull, St. Albans, UK), with measurements taken on the right-hand side of the body at two sites (triceps and subscapular). The median of three measurements at each site was used to estimate percent body fat (31). Lean body mass (LBM) was estimated as follows:

$$
\operatorname{LBM}(\mathrm{kg})=\text { body mass }(\mathrm{kg}) \times[1-(\% \mathrm{BF} / 100)]
$$


Paragraph 8 Participants provided a self-assessment of their level of physical maturity using drawings depicting the five stages of breast and pubic hair development (32). The scale ranges from 1 indicating pre-pubescent to 5 indicating full sexual maturation and participants were required to select the stage most closely resembling their current level of sexual development.

\section{Preliminary exercise measurements}

Paragraph 9 During the first visit to the laboratory, participants were familiarised with walking and running on the treadmill (Technogym Runrace, Gambettola, Italy) prior to completing two preliminary exercise measurements. The first test involved a $16 \mathrm{~min}$ incremental treadmill protocol divided into $4 \times 4$ min stages. The treadmill started at a speed of $4 \mathrm{~km} \cdot \mathrm{h}^{-1}$ and increased $1 \mathrm{~km} \cdot \mathrm{h}^{-1}$ at the start of each subsequent stage, with the gradient set at $1 \%$ throughout (19). This enabled the individual steady-state relationship between treadmill speed, oxygen uptake ( $\dot{\mathrm{VO}}_{2}$ ) and heart rate to be established. Participants were given a standardised 10 min passive rest period before completing the second exercise measurement to determine peak $\dot{\mathrm{V}}{ }_{2}$. The girls ran at a fixed individual speed $\left(6.8-9.5 \mathrm{~km} \cdot \mathrm{h}^{-}\right.$

${ }^{1}$ ), identified from the incremental treadmill protocol, while the treadmill gradient was increased $1 \%$ each minute until volitional exhaustion. Throughout both tests, heart rate was monitored continuously via short-range telemetry (PE4000, Polar-Electro, Kempele, Finland) and Borg's ratings of perceived exertion were recorded at standardised intervals (5). Expired air samples were monitored continuously using an online breath-by-breath gas analysis system (Cosmed $\mathrm{K} 4 \mathrm{~b}^{2}$, Rome, Italy), which was fully calibrated according to the manufacturer’s instructions before each use. Participants wore an appropriate size facemask (Hans Rudolf, Shawnee, USA), which was checked for leaks and connected to the online system via a flowmeter before each exercise test began. At least two of the following criteria 
were satisfied by all participants during the peak $\dot{\mathrm{V}} \mathrm{O}_{2}$ test to confirm maximal effort was achieved: a plateau in $\dot{\mathrm{V}}{ }_{2}(\leq 3 \%)$ with an increase in treadmill gradient; a peak heart rate $\geq$ 95\% of age-predicted maximum (220-chronological age); and a respiratory exchange ratio $\geq$ 1.10. An average of the breath-by-breath $\dot{\mathrm{VO}}_{2}$ data was taken every $10 \mathrm{~s}$, and peak $\dot{\mathrm{V}}_{2}$ was defined as the highest $30 \mathrm{~s}$ rolling average. Data from the incremental and peak $\dot{\mathrm{V}} \mathrm{O}_{2}$ protocols were used to determine the treadmill speed required to elicit $\sim 55 \%$ peak $\dot{\mathrm{V}}{ }_{2}$ during the main experimental exercise conditions.

\section{Experimental design}

Paragraph 10 Similar to previous studies with boys (2, 30, 34, 36), a within-measures, counterbalanced crossover design was adopted whereby participants completed three, two day experimental conditions: resting control (CON), 30 min intermittent treadmill exercise (EX30) and 60 min intermittent treadmill exercise (EX60). The conditions were separated by a standardised period of 14 days. The study design is shown schematically in Figure 1.

Day 1

Paragraph 11 The girls arrived at the laboratory at 15:30 and all measures were completed by 17:30. Body mass was recorded at the start of each experimental condition to standardise the test breakfast provided on Day 2 (below). During CON, participants rested for 110 min in the laboratory. During the exercise conditions, participants completed either 30 min (EX30) or $60 \mathrm{~min}$ (EX60) of moderate intensity intermittent treadmill exercise designed to elicit $\sim 55 \%$ peak $\dot{\mathrm{VO}} 2$. The exercise conditions were completed in either 3 x 10 min or 6 x $10 \mathrm{~min}$ intervals with a standardised 10 min period of passive rest between each interval. Oxygen uptake and heart rate were monitored continuously throughout each 10 min exercise interval 
as described previously and the treadmill speed was adjusted accordingly to ensure the target exercise intensity was achieved. Participants provided RPE at standardised intervals as described previously. The exercise energy expenditure and the oxidation rate of carbohydrate and fat were estimated via indirect calorimetry (10), assuming that participants reached a physiological steady state and that the urinary nitrogen excretion rate was negligible.

Day 2

Paragraph 12 Following a 12 h overnight fast, the girls arrived at the laboratory at 07:45 and a fasting capillary blood sample was taken after 10 min seated rest. A high-fat milkshake was consumed for breakfast within 10 min, marking the start of the postprandial period (08:00), and six further capillary blood samples were taken at hourly intervals throughout the $6 \mathrm{~h}$ postprandial period (Figure 1). Participants rested throughout the postprandial period and were able to read, watch DVD films and play non-active computer games. Participants consumed water ad libitum during the first condition and replicated the ingested volume during the subsequent conditions.

Standardisation of diet, physical activity and test milkshake

Paragraph 13 Participants recorded their dietary intake and all physical activity categorised according to intensity level in a detailed diary during the $48 \mathrm{~h}$ period preceding Day 2 of the first experimental condition. They were also asked to minimise their physical activity during this period and to replicate this diet and physical activity pattern before the second and third experimental conditions, which was confirmed verbally by the lead investigator. The overnight fasting period was standardised by asking participants to consume a small carbohydrate snack at 19:45 on Day 1 of each experimental condition (Kellogg's ${ }^{\circledR}$ nutrigrain strawberry 502 kJ, 3 g fat, 24 g carbohydrate, 2 g protein). After 20:00 the participants 
were allowed to drink plain water, but were asked not to consume any other drinks or food before arriving at the laboratory on Day 2.

Paragraph 14 The milkshake provided for breakfast on Day 2 contained vanilla dairy ice cream and double cream, at a ratio of 3:1, with $10 \mathrm{~g}$ of either powdered strawberry or chocolate flavour added to ensure the milkshake was palatable. Participants consumed the same flavour milkshake on all visits for consistency. The milkshake quantity was adjusted relative to body mass so that it provided $1.50 \mathrm{~g}$ of fat (70\% of total energy), $1.20 \mathrm{~g}$ of carbohydrate (25\%), $0.21 \mathrm{~g}$ of protein (5\%), and $80 \mathrm{~kJ}$ per kilogram body mass (34).

\section{Analytical methods}

Paragraph 15 After the hand was pre-warmed in water heated to $40^{\circ} \mathrm{C}$ for $5 \mathrm{~min}$, the fingertip was pierced (Unistick 3 Extra, Owen Mumford, UK), and between 300 and $600 \mu \mathrm{L}$ of whole capillary blood was collected into potassium-EDTA coated Microvette CB 300 tubes (Sarstedt Ltd, Leicester, UK). The whole blood samples were centrifuged immediately for 15 min at 12,800 g (Eppendorf 5415c, Hamburg, Germany). An automatic pipette was used to dispense $20 \mu \mathrm{L}$ of plasma into a $1.5 \mathrm{ml}$ Eppendorf tube (Fisher Scientific Ltd, Loughborough, UK), which was then diluted 50 times by adding $980 \mu \mathrm{L}$ of ice-cold saline (0.9\% NaCl) (2) (Hamilton Microlab 500 series, Reno, Nevada). This procedure was repeated so that two aliquots of diluted plasma were stored at $-80^{\circ} \mathrm{C}$ for up to two months before subsequent analysis (34). All samples were analysed for [TAG] and glucose concentration ([glucose]) by enzymatic, colorimetric methods (Randox Laboratories Ltd, Crumlin, UK) using a centrifugal analyser (Cobas Mira Plus, Roche, Basel, Switzerland). To account for the predilution of the plasma samples, the dilution step of the assay was eliminated, and three times the sample volume stated in the Randox kit assay procedure was used to ensure the 
concentration of the sample for analysis was the same as the original assay procedure $(2,34$, 36). The within-batch coefficients of variation for [TAG] and [glucose] were 3.4 and $2.2 \%$ respectively. Haemoglobin concentration and haematocrit were also quantified in the fasting and postprandial samples to estimate the acute change in plasma volume (9). Haemoglobin was measured in duplicate by haemophotometry (HemoCue AB, Ängelholm, Sweden) and haematocrit was assessed in duplicate using a microhaematocrit centrifuge and reader (Haematospin 1300 Microcentrifuge, Hawksley \& Sons Ltd, Sussex, UK).

\section{Statistical analyses}

Paragraph 16 Data were stored and analysed using the IBM SPSS statistics software for Windows version 19 (IBM corporation, New York, USA). Descriptive statistics outlining the physical and physiological characteristics of participants were determined. Normality of the data was confirmed by Shapiro Wilk tests and homogeneity of variances was checked by Mauchly's test of sphericity, with a Greenhouse-Geisser correction of the degrees of freedom applied if sphericity was violated. Student's paired t-tests were used to identify differences between the EX30 and EX60 responses. The total $6 \mathrm{~h}$ area under the plasma concentration versus time curves for TAG (TAUC-TAG) and glucose (TAUC-glucose) were calculated using the trapezium rule. Incremental versions of these were also calculated after accounting for respective fasting concentrations (iAUC-TAG and iAUC-glucose) across the conditions. The AUC responses, fasting blood concentrations and estimated changes in plasma volume were compared using separate one-way within-measures ANOVA. Differences in postprandial [TAG] and [glucose] were identified using separate $3 \times 7$ (condition by time) within-measures ANOVA. The 95\% confidence intervals (95\% CI) for mean absolute pairwise differences between experimental conditions were calculated using the t-distribution and degrees of freedom ( $n-1)$ and absolute standardised effect sizes (ES) are provided to 
supplement the findings. In the absence of a clinical anchor, an ES of 0.2 was considered the minimum important difference for all outcome measures, 0.5 moderate and 0.8 large (6). Bivariate correlations identifying possible determinants of the exercise-induced changes in TAUC-TAG were quantified using linear regression. Interpretation of the data will be based on $95 \%$ CI and ES.

\section{Results}

Responses to treadmill exercise

Paragraph 17 The treadmill responses for EX30 and EX60 are shown in Table 2. The girls exercised at an average intensity of 55\% and 56\% in EX30 and EX60 respectively (95\% CI 0.2 to $2.0 \%$, ES = 0.30). As expected, the exercise energy expenditure (95\% CI 686 to 832 $\mathrm{kJ}, \mathrm{ES}=3.59)$ and absolute fat (95\% CI 8 to $13 \mathrm{~g}, \mathrm{ES}=2.42)$ and carbohydrate oxidation (95\% CI 16 to $28 \mathrm{~g}, \mathrm{ES}=1.95$ ) were greater for EX60 compared with EX30. The only other difference in the exercise responses was a two unit increase in RPE for EX60 compared with EX30 (95\% CI 1 to 2, ES = 0.69).

Plasma volume changes and fasting [TAG] and [glucose]

Paragraph 18 Average changes in plasma volume between the fasting and 6 h postprandial samples were small between the conditions (CON -1.1\%, EX30 -2.4\%, EX60 -1.8\%; ES = 0.10 to 0.18 ). Therefore, the raw plasma [TAG] and [glucose] were not adjusted before statistical analyses. The fasting plasma [TAG] and [glucose] for each condition are displayed in Table 3. Fasting plasma [TAG] was lower after EX60 compared with CON (95\% CI -0.36 to $\left.0.04 \mathrm{mmol} \cdot \mathrm{L}^{-1}, \mathrm{ES}=0.41\right)$ and $\mathrm{EX} 30\left(95 \% \mathrm{CI}-0.47\right.$ to $0.04 \mathrm{mmol} \cdot \mathrm{L}^{-1}$, $\left.\mathrm{ES}=0.46\right)$, with no difference observed between EX30 and CON (95\% CI -0.22 to $0.34 \mathrm{mmol} \cdot \mathrm{L}^{-1}$, ES = 0.13). 
However, all group mean concentrations were low $\left(\leq 0.90 \mathrm{mmol} \cdot \mathrm{L}^{-1}\right)$. Differences in fasting plasma [glucose] between the three conditions were trivial ( $\mathrm{ES}=0.02$ to 0.21 ).

Plasma [TAG] and [glucose] in the postprandial period

Paragraph 19 Plasma [TAG] responses over the postprandial period for each experimental condition are shown in Figure 2. Differences in postprandial plasma [TAG] across the conditions were evident (main effect condition $\mathrm{ES}=0.36$, main effect time $\mathrm{ES}=0.39$, condition by time interaction ES $=0.10$ ). The TAUC-TAG (Table 3) was lower after EX60 compared with CON (95\% CI -2.66 to $\left.-0.04 \mathrm{mmol} \cdot \mathrm{L}^{-1} 6 \mathrm{~h}, \mathrm{ES}=0.40\right)$ and EX30 (95\% CI 2.11 to $\left.0.15 \mathrm{mmol} \cdot \mathrm{L}^{-1} 6 \mathrm{~h}, \mathrm{ES}=0.30\right)$; EX30 and CON were similar $(95 \% \mathrm{CI}-1.44$ to 0.71 $\mathrm{mmol} \cdot \mathrm{L}^{-1} 6 \mathrm{~h}, \mathrm{ES}=0.10$ ). Individual changes (delta) in TAUC-TAG between the exercise conditions and CON are shown in Figure 3. The reductions in TAUC-TAG following EX30 and EX60 were greater than changes in CON for ten ( 56\%) and twelve (67\%) girls respectively. Of the twelve girls that responded to EX60, nine also experienced a lower postprandial TAG response following EX30 compared with CON (data not shown). The Pearson's product moment correlation for the individual changes in TAUC-TAG between EX30 and EX60 revealed a moderate effect ( $r=0.57)$. The measured physical and physiological characteristics (Table 1) and exercise responses (Table 2) were not meaningfully correlated with the delta TAUC-TAG values for EX30 and EX60. The differences in iAUC-TAG between EX30 and the other two conditions were trivial (ES $\leq$ 0.15) and only a small effect was seen between EX60 and CON (ES = 0.30), although the 95\% CI suggested this was unlikely to be meaningful (-1.26 to $\left.0.33 \mathrm{mmol} \cdot \mathrm{L}^{-1} 6 \mathrm{~h}\right)$.

Paragraph 20 There was no difference in postprandial plasma [glucose] across the conditions (main effect condition ES =0.06; main effect time ES $=0.50$; condition by time 
interaction ES = 0.09). Similarly, there was little difference in either TAUC-glucose (ES = 0.05 to 0.13 ) or iAUC-glucose (ES = 0.08 to 0.22 ) between the conditions.

\section{Discussion}

Paragraph 21 The main finding of the present study was that 60, but not $30 \mathrm{~min}$, of moderate intensity treadmill exercise reduced postprandial [TAG] in healthy girls. Therefore, provided the energy expenditure is sufficient, performing moderate intensity exercise may represent an effective intervention to improve this aspect of cardiovascular health from a young age.

Paragraph 22 In the present study, a small to moderate reduction in fasting [TAG] was evident in EX60 compared with both CON and EX30. Although this largely supports previous findings in boys where the exercise gross EE was between 1.0 and $2.2 \mathrm{MJ}$ (2, 34, 36), fasting [TAG] are highly variable in children (35), and postprandial [TAG] are more predictive of cardiovascular disease risk than traditional fasting concentrations (1).

Paragraph 23 It is well established that a single session of moderate intensity exercise reduces postprandial [TAG] in adults (28). Furthermore, accumulating evidence demonstrates that moderate to vigorous intensity exercise promotes favourable changes in postprandial [TAG] in boys $(2,21,30,34,36)$, but no studies are available with girls. The reduction in postprandial [TAG] following EX60 supports previous studies in boys adopting a similar duration of moderate intensity exercise and inducing an exercise gross EE between 1.5 and $2.5 \mathrm{MJ}(2,21,30,34,36)$. The magnitude of change in postprandial lipaemia after $60 \mathrm{~min}$ moderate intensity exercise is highly variable in these studies, shown by the range of estimated ES between 0.32 to $0.86(2,21,30,34,36)$. Nevertheless, the moderate reduction seen in the present study is comparable to the average attenuation reported in the other 
studies with boys. In contrast to the present study, despite a similar doubling of the exercise gross EE in the 60 min $(1967 \mathrm{~kJ})$ compared with the 30 min condition $(982 \mathrm{~kJ})$, the reduction in postprandial [TAG] was similar following both exercise conditions in healthy boys (34). The lower exercise gross EE in this study is likely to reflect sex based differences in body composition and peak $\dot{\mathrm{VO}}_{2}$ (33). Although the TAG lowering effect of moderate intensity exercise may be determined by the exercise EE in adults (12, 37), this relationship has not been supported in healthy boys $(34,36)$. In the present study, the gross $\mathrm{EE}$ in the $60 \mathrm{~min}$ condition was approximately double that in the 30 min condition; however, only EX60 reduced postprandial [TAG]. A dose-response is not supported directly by these results, but given some of the girls experienced a reduction in EX30 TAUC compared with CON, it cannot by discounted completely. Further examination of the data did not reveal a "threshold" exercise EE suggested in our previous study (34) and elsewhere in the literature (24).

Paragraph 24 The 60 min exercise condition meets the minimum recommended level of physical activity to promote and maintain health in children and adolescents $(8,17)$. Although EX30 did not alter the postprandial TAG response systematically, the lowest dose of moderate intensity exercise EE required to reduce postprandial lipaemia consistently in girls is likely to be between 777 and $1536 \mathrm{~kJ}$, which is lower than the 2 MJ threshold suggested in adults (24). Although it is not possible to determine the clinical significance of our findings, the origin of atherosclerosis in childhood is well established (25), and cardiovascular risk factors are known to track from childhood to adulthood (3). It has been suggested that postprandial $[\mathrm{TAG}]<2.3 \mathrm{mmol} \cdot \mathrm{L}^{-1}$ in young people represents a healthy response (20), which is true of 95\% of the postprandial TAG samples in the present study. Therefore, the lower postprandial [TAG] after EX60 in girls with a predominantly healthy 
postprandial TAG profile is promising and supports the contention that interventions aimed at reducing cardiovascular risk factors should be initiated early in life (25).

Paragraph 25 Several studies have demonstrated that premenopausal women experience a lower postprandial TAG response than men $(7,16)$. Although the mechanisms responsible for this sex difference are not well established, it has been suggested that greater skeletal muscle uptake and retention of plasma [TAG], lower abdominal visceral adipose tissue lipolysis and greater suppression of upper-body subcutaneous adipose tissue lipolysis in women may be responsible $(7,15,18)$. However, it appears that men and women experience similar acute exercise-induced reductions in postprandial [TAG] (23.5 vs. 19.8\% respectively) (13). The moderate reduction in postprandial [TAG] following EX60 in the present study is comparable with the magnitude of change evident in similar studies with boys (2, 21, 30, 34, 36). Therefore, provided adequate energy is expended, healthy girls may acquire potential metabolic health benefits in postprandial TAG metabolism following moderate intensity exercise.

Paragraph 26 Considerable inter-individual variability was present in the postprandial TAG responses following the exercise conditions (Figure 3), with 'responders' and 'nonresponders' identified in both exercise conditions. Similar heterogeneity in the exerciseinduced changes in postprandial [TAG] has been reported with boys (34), and, consistent with the present study, the determinants of this variation could not be identified. In adults, changes in postprandial 3-hydroxybutyrate (3-OHB), a marker of hepatic fatty acid metabolism, accounted for almost half the variance evident in TAUC-TAG (11), and it is possible that this marker may explain some of the heterogeneity evident in our sample. Interestingly, a positive association was identified between the delta TAUC-TAG for EX30 and EX60, supporting previous findings with boys (34). This suggests that, at least at an 
individual level, girls demonstrating an attenuated postprandial TAG response after EX60 may also experience a reduction in postprandial [TAG] after EX30. It should be noted that the majority of girls in the current study self-classified themselves as mid to late pubertal. Although we found no effect of maturity status on any of the outcome measures or the degree of heterogeneity evident in postprandial [TAG], a possible maturation effect cannot be ruled out entirely.

Paragraph 27 The mechanisms underpinning the reduction in postprandial [TAG] after EX60 cannot be determined from our findings. However, in adults, it is proposed that a greater removal rate of circulating TAG facilitated by higher skeletal muscle lipoprotein lipase activity may be responsible (14) although other mechanisms were also suggested. An alternative mechanism implicates the diminished appearance of VLDL-TAG in the circulation (23), possibly due to the secretion of fewer, TAG-richer VLDL particles which are likely to have a greater affinity for lipoprotein lipase (22). Indirect evidence for altered hepatic VLDL secretion can be derived from a recent study, reporting that the exerciseinduced reduction in postprandial [TAG] is associated with the elevation in the concentration of postprandial 3-OHB (11). This suggests that exercise may result in a shift towards hepatic fatty acid oxidation and away from fatty acid re-esterification and VLDL-TAG synthesis (11).

Paragraph 28 In conclusion, 60 min of moderate intensity exercise, with a gross EE of 1536 $\mathrm{kJ}$ performed $\sim 14.5 \mathrm{~h}$ prior to a high fat breakfast, reduced postprandial [TAG] in healthy girls. Performing 30 min moderate intensity exercise, inducing half the exercise gross EE (777 kJ), was insufficient to reduce postprandial [TAG] in this population.

\section{Acknowledgements}


The authors thank Alsager High School, Outwoods Edge Primary School and Woodbrook Vale High School in Loughborough for their support and the participants and their parents for their commitment throughout this research. The authors declare no conflict of interest. No funding was received for this research, other than that available internally through Loughborough University. The results of the present study do not constitute endorsement by the American College of Sports Medicine. 


\section{References}

1. Bansal S, Buring JE, Rifai N, Mora S, Sacks FM, Ridker PM. Fasting compared with nonfasting triglycerides and risk of cardiovascular events in women. JAMA. 2007;298(3):309-16.

2. Barrett LA, Morris JG, Stensel DJ, Nevill ME. Exercise and postprandial plasma triacylglycerol concentrations in healthy adolescent boys. Med Sci Sports Exerc. 2007;39(1):116-22.

3. Berenson GS, Srinivasan SR, Bao W, Newman WP, Tracy RE, Wattigney WA. Association between multiple cardiovascular risk factors and atherosclerosis in children and young adults. The Bogalusa Heart Study. N Engl J Med. 1998;338(23):1650-6.

4. Boquist S, Ruotolo G, Tang R, et al. Alimentary lipemia, postprandial triglyceride-rich lipoproteins, and common carotid intima-media thickness in healthy, middle-aged men. Circulation. 1999;100(7):723-8.

5. Borg GAV, Noble BJ. Perceived exertion. Exerc Sport Sci Rev. 1974;2(1):131-53.

6. Cohen J. Statistical Power Analysis for the Behavioural Sciences. 2nd ed. Hillsdale (NJ): Lawrence Erlbaum Associates; 1988. 23-4 p.

7. Couillard C, Bergeron N, Prud'homme D, et al. Gender difference in postprandial lipemia : importance of visceral adipose tissue accumulation. Arterioscler Thromb Vasc Biol. 1999;19(10):2448-55.

8. Department of Health, Physical Activity, Health Improvement and Protection. Start active, stay active. A report on physical activity for health from the four home countries' 
Chief Medical Officers. London: United Kingdom Department of Health, Physical Activity, Health Improvement and Protection; 2011. 26 p. Available from UK Department of Health.

9. Dill DB, Costill DL. Calculation of percentage changes in volumes of blood, plasma, and red cells in dehydration. J Appl Physiol. 1974;37(2):247-8.

10. Frayn KN. Calculation of substrate oxidation rates in vivo from gaseous exchange. $J$ Appl Physiol. 1983;55(2):628-34.

11. Gill JMR, Al-Mamari A, Ferrell WR, et al. Effect of prior moderate exercise on postprandial metabolism in men with type 2 diabetes: heterogeneity of responses. Atherosclerosis. 2007;194(1):134-43.

12. Gill JMR, Herd SL, Hardman AE. Moderate exercise and post-prandial metabolism: issues of dose-response. J Sports Sci. 2002;20(12):961-7.

13. Gill JMR, Herd SL, Tsetsonis NV, Hardman AE. Are the reductions in triacylglycerol and insulin levels after exercise related? Clin Sci. 2002;102(2):223-31.

14. Gill JMR, Herd SL, Vora V, Hardman AE. Effects of a brisk walk on lipoprotein lipase activity and plasma triglyceride concentrations in the fasted and postprandial states. Eur J Appl Physiol. 2003;89(2):184-90.

15. Horton TJ, Commerford SR, Pagliassotti MJ, Bessesen DH. Postprandial leg uptake of triglyceride is greater in women than in men. Am J Physiol Endocrinol Metab. 2002;283(6):E1192-202. 
16. Jackson KG, Clarke DT, Murray P, et al. Introduction to the DISRUPT postprandial database: subjects, studies and methodologies. Genes Nutr. 2010;5(1):39-48.

17. Janssen I, LeBlanc AG. Systematic review of the health benefits of physical activity and fitness in school-aged children and youth. Int J Behav Nutr Phys Act. 2010;7:40.

18. Jensen MD. Gender differences in regional fatty acid metabolism before and after meal ingestion. J Clin Invest. 1995;96(5):2297-303.

19. Jones AM, Doust JH. A 1\% treadmill grade most accurately reflects the energetic cost of outdoor running. J Sports Sci. 1996;14(4):321-7.

20. Kolovou GD, Bilianou H, Mikhailidis DP. Postprandial Lipemia in Children and Adolescents. Curr Vasc Pharmacol. 2011;9(3):318-20.

21. MacEneaney OJ, Harrison M, O'Gorman DJ, Pankratieva EV, O'Connor PL, Moyna NM. Effect of prior exercise on postprandial lipemia and markers of inflammation and endothelial activation in normal weight and overweight adolescent boys. Eur J Appl Physiol. 2009;106(5):721-9.

22. Magkos F, Wright DC, Patterson BW, Mohammed BS, Mittendorfer B. Lipid metabolism response to a single, prolonged bout of endurance exercise in healthy young men. Am J Physiol Endocrinol Metab. 2006;290(2):E355-62.

23. Malkova D, Evans RD, Frayn KN, Humphreys SM, Jones PR, Hardman AE. Prior exercise and postprandial substrate extraction across the human leg. Am J Physiol Endocrinol Metab. 2000;279(5):E1020-8. 
24. Maraki M, Sidossis LS. Effects of energy balance on postprandial triacylglycerol metabolism. Curr Opin Clin Nutr Metab Care. 2010;13(6):608-17.

25. McGill HC, McMahan CA, Herderick EE, Malcom GT, Tracy RE, Strong JP. Origin of atherosclerosis in childhood and adolescence. Am J Clin Nutr. 2000;72(5):1307S-1315S.

26. Nader PR, Bradley RH, Houts RM, McRitchie SL, O'Brien M. Moderate-to-vigorous physical activity from ages 9 to 15 years. JAMA. 2008;300(3):295-305.

27. Nordestgaard BG, Benn M, Schnohr P, Tybjærg-Hansen A. Nonfasting triglycerides and risk of myocardial infarction, ischemic heart disease, and death in men and women. JAMA. 2007;298(3):299-308.

28. Peddie MC, Rehrer NJ, Perry TL. Physical activity and postprandial lipidemia: are energy expenditure and lipoprotein lipase activity the real modulators of the positive effect? Prog Lipid Res. 2012;51(1):11-22.

29. Riddoch CJ, Mattocks C, Deere K, et al. Objective measurement of levels and patterns of physical activity. Arch Dis Child. 2007;92(11):963-9.

30. Sedgwick MJ, Morris JG, Nevill ME, Tolfrey K, Nevill A, Barrett LA. Effect of exercise on postprandial endothelial function in adolescent boys. Br J Nutr. 2013;110(2):301-9.

31. Slaughter MH, Lohman TG, Boileau RA, et al. Skinfold equations for estimation of body fatness in children and youth. Hum Biol. 1988;60(5):709-23.

32. Tanner JM. Growth at adolescence. 2nd ed. Oxford, UK: Blackwell Scientific Publications; 1962. 28-39 p. 
33. Tarnopolsky LJ, MacDougall JD, Atkinson SA, Tarnopolsky MA, Sutton JR. Gender differences in substrate for endurance exercise. J Appl Physiol. 1990;68(1):302-8.

34. Tolfrey K, Bentley C, Goad M, Varley J, Willis S, Barrett L. Effect of energy expenditure on postprandial triacylglycerol in adolescent boys. Eur J Appl Physiol. 2012;112(1):23-31.

35. Tolfrey K, Campbell IG, Jones AM. Intra-individual variation of plasma lipids and lipoproteins in prepubescent children. Eur J Appl Physiol. 1999;79(5):449-56.

36. Tolfrey K, Doggett A, Boyd C, Pinner S, Sharples A, Barrett L. Postprandial triacylglycerol in adolescent boys: a case for moderate exercise. Med Sci Sports Exerc. 2008;40(6):1049-56.

37. Tsetsonis NV, Hardman AE. Effects of low and moderate intensity treadmill walking on postprandial lipaemia in healthy young adults. Eur J Appl Physiol. 1996;73(5):419-26.

38. Zilversmit DB. Atherogenesis: a postprandial phenomenon. Circulation. 1979;60(3):47385. 


\section{Figure legends}

Figure 1. Schematic of two-day study protocol. TAG, triacylglycerol; $C O N$, rest control condition; EX30, 30 min intermittent exercise condition; EX60, 60 min intermittent exercise condition. ${ }^{\dagger}$ Evening meal replicated from $1^{\text {st }}$ condition.

Figure 2. Fasting (F) and postprandial plasma triacylglycerol (TAG) concentration for the control condition (CON), and the $30 \mathrm{~min}$ (EX30), and 60 min (EX60) intermittent exercise conditions $(n=18)$. Black rectangle signifies consumption of the breakfast meal (08:00). Main effect for condition $(\mathrm{ES}=0.36)$; main effect for time $(\mathrm{ES}=$ 0.39); condition by time interaction $(\mathrm{ES}=0.10)$.

Figure 3. Individual changes (delta) in the total area under the plasma triacylglycerol (TAG) concentration versus time curve (TAUC) between the $30 \mathrm{~min}$ (EX30) and $60 \mathrm{~min}$ (EX60) intermittent exercise conditions and the rest control condition (CON): A) EX30 minus CON; B) EX60 minus CON. Participant data are ordered according to the size of the exercise-induced change in TAUC-TAG; thus, the order of the individual participants is not identical in A and B. A negative response indicates a reduction in TAUC-TAG in the exercise condition compared with CON. 